\title{
Aspergillus Infection Inhibits the Expression of Peanut 13S-HPODE-Forming Seed Lipoxygenases
}

\author{
Dimitrios I. Tsitsigiannis, ${ }^{1}$ Susan Kunze, ${ }^{2}$ David K. Willis, ${ }^{1,3}$ Ivo Feussner, ${ }^{2}$ and Nancy P. Keller ${ }^{1}$ \\ ${ }^{1}$ Department of Plant Pathology, University of Wisconsin-Madison, Madison 53706, U.S.A.; ${ }^{2}$ Georg-August-University \\ Göttingen, Albrecht-von-Haller Institute for Plant Sciences, Department for Plant Biochemistry, Justus-von-Liebig-Weg 11, \\ D-37077 Göttingen, Germany; ${ }^{3}$ United States Department of Agriculture-Agricultural Research Service Vegetable Crops \\ Research Unit, University of Wisconsin-Madison, Madison 53706, U.S.A.
}

Submitted 11 April 2005. Accepted 4 June 2005.

\begin{abstract}
Oxylipins recently have been implicated as signaling molecules for cross-kingdom communication in plant-pathogen interactions. Linoleic acid and its two plant lipoxygenase (LOX) oxylipin products 9- and 13-hydroperoxy fatty acids (9S- and 13S-HPODE) have been shown to have a significant effect on differentiation processes in the mycotoxigenic seed pathogens Aspergillus spp. Whereas both fatty acids promote sporulation, $9 S$-HPODE stimulates and $13 S$ HPODE inhibits mycotoxin production. Additionally, Aspergillus flavus infection of seed promotes linoleate 9-LOX expression and 9S-HPODE accumulation. Here, we describe the characterization of two peanut seed lipoxygenase alleles (PnLOX2 and PnLOX3) highly expressed in mature seed. PnLOX2 and PnLOX3 both are 13S-HPODE producers (linoleate 13-LOX) and, in contrast to previously characterized 9-LOX or mixed function $L O X$ genes, are repressed between 5 -fold and 250 -fold over the course of $A$. flavus infection. The results of these studies suggest that $9 S$ HPODE and 13S-HPODE molecules act as putative susceptibility and resistance factors respectively, in Aspergillus seed-aflatoxin interactions.
\end{abstract}

Additional keywords: Arachis hypogaea, defense.

Seed oxylipins (oxygenated polyenoic fatty acids) may be produced by plant lipoxygenases (LOXs) (EC 1.13.11.12), a functionally diverse class of nonheme dioxygenases implicated in physiological processes such as growth and development, seed germination, senescence, formation of flavor and aroma compounds, and stress- and pest-related responses (Farmer et al. 2003; Hildebrand et al. 1998; Howe and Schilmiller 2002; Porta and Rocha-Sosa 2002; Wasternack and Hause 2002). Distinct plant LOX isozymes preferentially introduce molecular oxygen into linoleic and linolenic acids either at C-9 (9LOX) or at C-13 (13-LOX) of the hydrocarbon backbone of the fatty acid to produce cis-trans $9 S$ - or $13 S$-hydroperoxy linoleic acid $(9 S$ - or $13 S$-HPODE) or $9 S$ - or $13 S$-hydroperoxy linolenic acid ( $9 S$ - or 13S-HPOTE). The 13-monohydroperoxides are precursors of biologically active compounds such as traumatin, jasmonic acid, and methyl jasmonate, which have hormone-like regulatory and defense-related roles in plants (Blee

Corresponding author: N. P. Keller; Telephone: +1-608-262-9795; Fax: +1-606-263-2626; E-mail: npk@plantpath.wisc.edu

Current address of D. I. Tsitsigiannis: The Sainsbury Laboratory, John Innes Centre, Norwich Research Park, Colney Lane, Norwich NR4 7UH, U.K.
2002; Feussner and Wasternack 2002). The 9-monohydroperoxides are converted into compounds whose physiological actions are not known, although there is evidence that some of them have antimicrobial properties and induce the hypersensitive reaction, a form of programmed cell death and one of the active defense resistance mechanisms against microbial invasion (Blee 2002; Feussner and Wasternack 2002; Gobel et al. 2003; Kolomiets et al. 2000). Antisense suppression of a potato $13-L O X$ isoform led to a plant deficient in proteinase accumulation, which was associated with increased weight gain of Colorado potato beetles fed on antisense plants compared with wild-type plants (Royo et al. 1999), and tobacco plants suppressed in 9-LOX expression became susceptible to Phytophthora parasitica infections (Rance et al. 1998).

Recent studies have implicated a role for oxylipins in signaling between kingdoms (Oh et al. 2001; Wang et al. 2004). A developing case for cross-kingdom communication in plantpathogen interactions occurs in the interaction between seed and mycotoxigenic Aspergillus spp. The filamentous fungi Aspergillus flavus and $A$. parasiticus colonize oil seed (e.g., corn, peanut, cotton, and nut) and cause tremendous yield and economic loss through tissue maceration, as well as a significant health problem by the contamination of the seed with the mycotoxin aflatoxin, the most potent natural carcinogen known (Bennett and Klich 2003; Cleveland et al. 2003). Linoleic acid and its two plant LOX products, $9 S$ - and $13 S$-HPODE, had a significant effect on differentiation processes in A. nidulans, A. flavus, and A. parasiticus. Whereas all of the $18 \mathrm{C}$ polyunsaturated fatty acids could promote sporulation in all three species, 9S-HPODE stimulated and 13S-HPODE inhibited mycotoxin production, presumably by structurally mimicking endogenous Aspergillus sporogenic factors: oxylipins derived from oleic, linoleic, and linolenic acid (Burow et al. 1997; Calvo et al. 1999; Tsitsigiannis et al. 2004a and b, 2005). Other LOX pathway metabolites (e.g., methyl jasmonate and aldehyde products of $13 S$-HPODE and 13S-HPOTE) also have been reported to inhibit or stimulate fungal development and aflatoxin production (Doehlert et al. 1993; Goodrich-Tanrikulu et al. 1995; Vergopoulou et al. 2001; Zeringue 1996).

The importance of the LOX pathway products in signaling Aspergillus developmental processes led us to further dissect the role of plant LOX in the Aspergillus-seed interaction. Previous studies in our laboratory have demonstrated that peanut seed and maize seed $L O X$ genes are induced by Aspergillus infection. In maize seed, Aspergillus infections induce expression of cssap 92 (9-LOX) in maize lines susceptible to aflatoxin contamination, but repress cssap 92 expression in lines resistant to aflatoxin production (Wilson et al. 2001). This ob- 
servation could indicate that cssap 92 is a biomarker denoting "resistance" or "susceptibility" to aflatoxin contamination in the field.

In peanut seed, Aspergillus infections induced expression of PnLOX1 encoding a mixed-function LOX producing approximately 21\% 9S-HPODE and 59\% 13S-HPODE (Burow et al. 2000). However, biochemical analysis of the infected seed-in contrast to noninfected seed-showed a steady increase in $9 S$ HPODE content of the seed during the course of Aspergillus colonization (Burow et al. 2000). These results led to the conclusion that additional peanut seed $L O X$ s, both 9 and 13-LOX, actively participate in the seed-Aspergillus interaction where 9-LOX gene expression would be induced and 13-LOX expression possibly repressed during fungal infection. Here, we characterize two peanut seed $L O X$ alleles (PnLOX2 and PnLOX3) that are strongly repressed after $A$. flavus infection. PnLOX2 and PnLOX3 are both 13S-HPODE producers (13-LOX) and are specifically expressed in seed. These studies further support a case for 9-LOX as susceptibility factors and 13-LOX as resistance factors in mycotoxin contamination in seed crops.

\section{RESULTS}

$P n L O X 2$ and PnLOX3 gene isolation and characterization.

To identify and characterize peanut seed $L O X$ genes at the molecular and biochemical level, we performed an alignment analysis of several amino acid sequences of known $L O X$ genes from other plant species. Degenerate primers were designed over conserved areas, and polymerase chain reaction (PCR) products (approximately $1.5-\mathrm{kb}$ ) were amplified from peanut seed cDNA pools created from seed infected by A. flavus for 8 and $48 \mathrm{~h}$ and mock-inoculated (control) peanut seed and cloned into plasmid pBSK. Restriction fragment polymorphism analysis of the 130 clones identified four different restriction fragment length polymorphism groups from control seed and five groups from infected seed, where groups A and B were found in both control and infected seed (Table 1). Clones of group A showed the same digestion patterns as the previously characterized PnLOXI (Burow et al. 2000). Representative clones from all groups were end sequenced and aligned with $P n L O X 1$. The results revealed a high degree of identity, a common feature for genes of the $L O X$ family. Specific primers were designed from the end sequences to obtain, by $5^{\prime}$ and $3^{\prime}$ rapid amplification of cDNA ends (RACE), full-length cDNAs of putative novel peanut $L O X$ genes. Full-length sequences were obtained successfully from group B (control-PnLOX2) and group $\mathrm{C}$ (infected-PnLOX3) seed cDNA pools. The full- length cDNA of PnLOX2 (accession number DQ068249) has 2,803 nucleotides and encodes 863 amino acid residues, whereas the full-length cDNA of PnLOX3 (accession number DQ068250) has 2,864 nucleotides and a longer 5' untranslated region, but also encodes 863 amino acid residues. The deduced amino acid sequence of PnLOX2 and PnLOX3 (we will refer to both of them as PnLOX2-3 for the rest of this article) showed 99\% identity to each other and 91 and $92 \%$ identity, respectively, to PnLOX1. PnLOX2-3 had significant structural identity with several other plant LOXs, ranging from 70 to $80 \%$ identity with legume LOXs and 55 to $60 \%$ with potato, tomato, and Arabidopsis spp. Additionally, phylogenetic analysis of different plant LOX proteins showed that PnLOX2 and PnLOX3 are grouped within the closely related cluster of legume LOXs (Fig. 1) and are classified into the type I 13LOXs, the gene subfamily of 13-LOXs that does not harbor a transit peptide (type II 13-LOXs carry a putative chloroplast transit peptide sequence) (Feussner and Wasternack 2002).

Conserved domain search of the PnLOX2-3 proteins using NCBI Conserved Domain Search analysis indicated that the residues 1 to 120 had domains similar to PLAT (polycystin-1, lipoxygenase, $\alpha$-toxin; cd00113.1) domain or LH2 (lipoxygenase homology; smart00308.10) domain that consists of an eight-stranded $\beta$-barrel. This domain is found at the $\mathrm{N}$ terminus in lipoxygenases and at the $C$ terminus in lipases; it is required for the enzymes to access their sequestered membrane or micelle-bound substrates by mediating attachment via other protein-binding partners. PSORT analysis also indicated that both proteins lack the chloroplast targeting motifs found in many other plant LOXs and, therefore, were predicted to be cytoplasmic. Additionally, the deduced amino acid sequences of PnLOX2-3 contained the conserved four histidine residues postulated to be important for enzyme activity of plant LOXs binding to iron cofactors (Steczko et al. 1992).

\section{Biochemical properties \\ of recombinant PnLOX2 and PnLOX3.}

In order to estimate the biochemical properties of the LOXs from peanut, both cDNAs were expressed in a bacterial system and analyzed for their $\mathrm{pH}$ optima and product specificities against linoleic acid. Both enzymes showed nearly identical $\mathrm{pH}$ optima and regio specificities. They turned out to be active only at neutral to alkaline $\mathrm{pH}$ values above $\mathrm{pH}$ 7.7. In contrast, previous studies indicated that the PnLOX1 enzyme is active at pH 6, 6.5, and 7 (Burow et al. 2000). The regio specificities against linoleic acid were in favor of $13 S$-HPODE, thus identifying both enzymes as linoleate 13-LOXs (Table 2). In contrast

Table 1. Restriction fragment length polymorphism (RFLP) analysis results of lipoxygenase polymerase chain reaction (PCR) products ${ }^{\mathrm{a}}$

\begin{tabular}{llllllcc}
\hline Group & A & B & C & D & E & F & G \\
\hline Infected & 38 & 15 & 15 & 23 & 9 & $\ldots$ & $\ldots$ \\
Control & 34 & 64 & $\ldots$ & $\ldots$ & $\ldots$ & 1 & 1 \\
\hline
\end{tabular}

${ }^{a}$ Collection of cloned PCR products amplified with degenerative primers from peanut seed infected or mock inoculated (control) with Aspergillus flavus were digested with Sau3A1 or AluI. The resulting restriction patterns sharing similar RFLP profiles were grouped into seven categories (A to G). Numbers indicate percentage (\%) of clones per group.

Table 2. Analyses of the oxylipin products of the reaction of PnLOX2 and PnLOX3 with linoleic acid at $\mathrm{pH} 8.0^{\mathrm{a}}$

\begin{tabular}{lcccc}
\hline & Isomers & & \multicolumn{2}{c}{ Optical isomers (S:R) } \\
\cline { 2 - 3 } LOX forms & 13ZE-HOD:13EE-HOD:9EZ-HOD:9EE-HOD & & 13-ZE-HOD & 9-EZ-HOD \\
\hline PnLOX2 & $78: 5: 15: 2$ & $95: 5$ & $70: 30$ \\
PnLOX3 & $80: 4: 15: 1$ & $96: 4$ & $78: 22$ \\
\hline
\end{tabular}

${ }^{\text {a }}$ LOX $=$ lipoxygenase andHOD $=$ hydroxy-octadecadienoic acid. Oxygenated linoleic acid derivatives were isolated and isomers were given as molar ratios, as determined by straight-phase high-performance liquid chromatography (SP-HPLC). Molar ratios of S and R optical isomers were determined by CPHPLC. The data represent the mean values of three measurements from two independent experiments. 


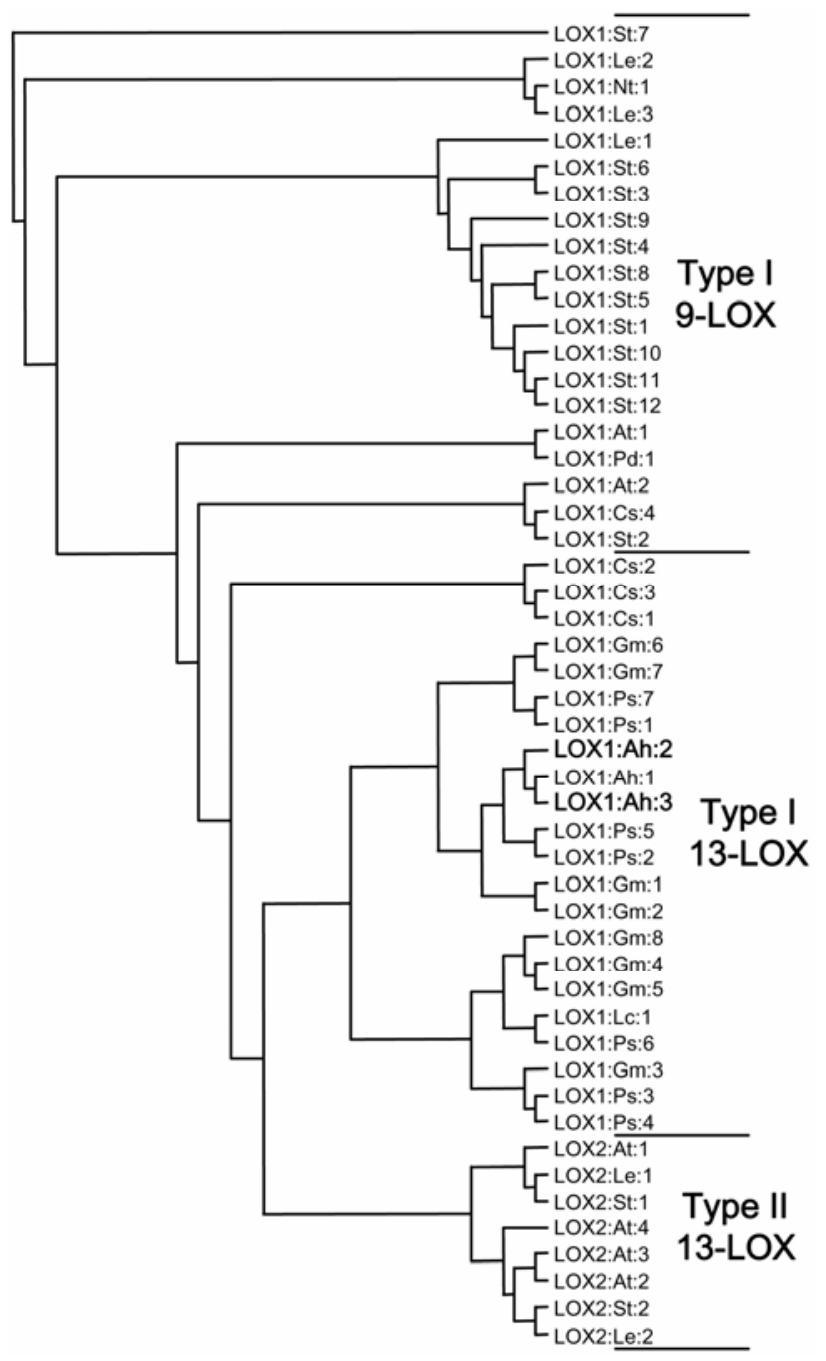

Fig. 1. Comparison of the PnLOX2 and PnLOX3 with the deduced aminoacid sequences of different plant lipoxygenases (LOXs) in a phylogenetic tree analysis. The tree was compiled using the PHYLIP (Phylogeny Inference Package, version 3.63) program. The proteins mentioned in the tree refer to the corresponding accession numbers in the GenBank. Sequences from distinct plant species have been included and have been partially renamed according to the nomenclature of Shibata and associates (1994): Arachis hypogaea: LOX1:Ah:1 (AAF60270), LOX1:Ah:2 (DQ068249), LOX1:Ah:3 (DQ068250); Arabidopsis thaliana: LOX1:At:1 (AtLOX1, AAA32827), LOX2:At:1 (AtLOX2, AAA32749), LOX2:At:2 (AtLOX3, AAF79461), LOX2:At:3 (AtLOX4, AAF21176), LOX1:At:2 (AtLOX5, CAC19365), LOX2:At:4 (AtLOX6, AAG52309); Cucumis sativus: LOX1:Cs:1 (AAC61785), LOX1:Cs:2 (CsULOX, AAA79186), LOX1:Cs:3 (CsLBLOX, CAA63483), LOX1:Cs:4 (CAB83038); Glycine max: LOX1:Gm:1 (soybean LOX1, AAA33986), LOX1:Gm:2 (soybean LOX2, AAA33987), LOX1:Gm:3 (soybean LOX3, CAA31664), LOX1:Gm:4 (soybean vlxa, BAA03101), LOX1:Gm:5 (soybean vlxb, AAB67732), LOX1:Gm:6 (soybean vlxc, AAA96817), LOX1:Gm:7 (soybean vlxd, AAA03728), LOX1:Gm:8 (soybean vlxe, AAC49159); Lycopersicon esculentum: LOX1:Le:1 (tomLOXA, P38415, AAA53184), LOX1:Le:2 (tomLOXB, AAA53183), LOX1:Le:3 (tomLOX-tox, AAG21691), LOX2:Le:1 (tomLOXC, AAB65766), LOX2:Le:2 (tomLOXD, AAB65767); Nicotiana tabacum: LOX1:Nt:1 (NtLOX, CAA58859); Pisum sativum: LOX1:Ps:1 (AAB71759), LOX1:Ps:2 (CAA55318), LOX1:Ps:3 (CAA55319), LOX1:Ps:4 (Pea LOX, CAA30666), LOX1:Ps:5 (Pea LOX2, CAA34906), LOX1:Ps:6 (Pea LOXG, CAA53730), LOX1:Ps:7 (CAC04380); Prunus dulchis: LOX1:Pd:1 (CAB94852); Solanum tuberosum: LOX1:St:1 (SOLTULOX1, CAA55724), LOX1:St:2 (STLOX, AAD09202), LOX1:St:3 (StLOX1, S73865 AAB31252), LOX1:St:4 (CAA64766), LOX1:St:5 (CAA64765), LOX1:St:6 (POTLX-2, AAB67860), LOX1:St:7 (POTLX-3, AAB67865), LOX1:St:8 (POTLX-1, AAB67858), LOX1:St:9 (AAD04258), LOX1:St:10 (pLOX2, AAB81595), LOX1:St:11 (pLOX1, AAB81594), LOX1:St:12 (CAB65460), LOX2:St:1 (StLOXH1, CAA65268), LOX2:St:2 (StLOXH3, CAA65269). to PnLOX1, the amount of $13 S$-HPODE formed was 78 and $80 \%$ for PnLOX2 and PnLOX3, respectively. These data may identify both LOXs as pure 13-LOX, whereas PnLOX1 lacks pronounced region specificity.

\section{PnLOX2 and PnLOX3 are expressed in seed.}

To determine the organ-specific expression pattern of PnLOX2-3 in healthy plants, quantitative reverse-transcription (qRT)-PCR was performed on RNA isolated from lateral roots, taproot, peg, immature 1- and 2-week-old seed, immature pod shell, stem, leaves, flowers, mature cotyledon, embryo, and green cotyledon after seedling emergence. The expression level of PnLOX2-3 was very low in flower tissues and this value was used as the calibrator condition for comparison with other organs and relative to the housekeeping gene actin-DF. Based on the data in Table 3, PnLOX2-3 expression appears to be seed specific and expression is highest in mature embryo and immature cotyledons. Low expression is observed in the shell of the pods and at almost undetectable levels in other organs.

\section{PnLOX2, PnLOX3, or both are repressed during Aspergillus infection of mature seed.}

Expression of PnLOX2-3 was examined after wounding and infection of mature seed, conditions commonly found to induce expression of other LOX genes, including PnLOXI (Bell and Mullet 1993; Burow et al. 2000; Choi et al. 1994; Howe and Schilmiller 2002). Real-time qRT-PCR analysis of the transcriptional kinetics of PnLOX2-3 that occurs during A. flavus colonization compared with mock inoculation and wounding is shown in Figure 2A. The calibrator for this experiment was the average of normalized transcript from mock-inoculated cotyledons. The expression of PnLOX2-3 was stable for all treatments at $0 \mathrm{~h}$ and was downregulated at 6 and $12 \mathrm{~h}$ post inoculation. At $18 \mathrm{~h}$ and beyond, transcription increased over time, reaching an approximately 65 - and 79-fold increase for the mock-inoculated and wounding samples, respectively, $96 \mathrm{~h}$ after inoculation. In sharp contrast, A. flavus infection repressed the expression of PnLOX2-3 24 h post inoculation, a time that coincides with the first development of visible symptoms and fungal growth in planta. Repression ranged between 5- and 250 -fold over the course of infection compared with control and wounding treatments. Experiments were repeated three independent times for several timepoints with similar results and additionally were confirmed by Northern analysis (Fig. 2B). These data indicate that in, contrast to $P n L O X 1$, which is

Table 3. Expression of PnLOX2-3 in peanut organs measured by qRT-PCR analysis $^{\mathrm{a}}$

\begin{tabular}{lrr}
\hline Peanut organs & $\begin{array}{c}\text { Relative expression } \\
\text { ratio }^{\mathbf{b}}\end{array}$ & $\begin{array}{c}\text { Standard } \\
\text { error }\end{array}$ \\
\hline Flowers & 1.0 & 0.1 \\
One-week-old seed & 5.6 & 0.2 \\
Three-week-old seed & $21,480.1$ & $1,280.1$ \\
Four-week-old immature shell & 79.0 & 24.4 \\
Mature cotyledon & $1,882.5$ & 140.7 \\
Embryo & $21,607.1$ & $1,697.8$ \\
Germinated cotyledon & 37.2 & 1.7 \\
Tap root & 0.1 & 0.05 \\
Lateral root & 2.5 & 0.3 \\
Peg & 13.6 & 4.1 \\
Stem & 5.8 & 0.7 \\
Leaf & 0.3 & 0.1
\end{tabular}

${ }^{a}$ qRT-PCR = quantitative reverse-transcriptase polymerase chain reaction.

$\mathrm{b}$ The amount of lipoxygenase ( $L O X)$ expression at each organ first was normalized to the internal standard RNA (actin); then, the normalized $L O X$ ratio value was divided by calibrator consisting of the average of the normalized $L O X$ values in flowers. 
upregulated after Aspergillus infection (Burow et al. 2000), expression of $P n L O X 2-3$ is significantly repressed by the pathogen.

\section{DISCUSSION}

In plants, LOX metabolites are converted into a wide array of compounds having functions that range from elicitation of plant defenses to generation of volatiles that are released in response to insect feeding and serve as a chemical signal for natural insect enemies (Jalloul et al. 2002; Rance et al. 1998; Royo et al. 1999; Rusterucci et al. 1999). Each LOX produces different ratios of 13- and 9-HPODEs that are metabolized by different pathways to yield various oxylipin products (Feussner and Wasternack 2002), some of which activate or repress Aspergillus differentiation processes, including spore development, sclerotial development, and mycotoxin biosynthesis (Burow et al. 1997; Calvo et al. 1999; Wright et al. 2000; Zeringue 1996). The purpose of this study was to further explore the hypothesis that $9 S$-HPODE stimulation of aflatoxin production was associated with 9-LOX induction or 13-LOX repression in susceptible seed. Our previous studies supported the case that 9- $L O X$ or mixed function $L O X$ genes are activated during $A s$ pergillus colonization; however, the response of 13-LOX genes to Aspergillus infections had not been investigated.
Previous biochemical studies led to the identification of at least three different isozymes in peanut seed, where one produced predominantly $9 S$-HPODE, one $13 S$-HPODE, and one a mixture of 9- and 13-HPODEs (Pattee and Singleton 1977; Sanders et al. 1975). Our previous work identified a mixedfunction peanut LOX, PnLOX1 (21\% $9 S$ - and 59\% $13 S$ HPODE) that was activated by wounding and Aspergillus infections (Burow et al. 2000). However, biochemical data indicated that additional LOX genes were involved in the Aspergillus spp.-peanut interaction because $9 S$-HPODE levels increased significantly in infected seed (from $7.2 \%$ at $8 \mathrm{~h}$ to $35.6 \%$ at 48 $\mathrm{h}$ post infection), whereas $13 \mathrm{~S}$-HPODE remained the dominant metabolite in mock-inoculated seed ( 80 to $83 \%$ from 8 to $48 \mathrm{~h}$ ) but repressed in infected seed, from $78 \%$ at $8 \mathrm{~h}$ to $41 \%$ at $48 \mathrm{~h}$ after Aspergillus colonization (Burow et al. 2000), a timepoint that leads to high levels of aflatoxin production (Lopez et al. 1998). The changes in oxylipin accumulation under these conditions suggested that 9-LOX was induced and 13- $L O X$ repressed in the infected seed. Here, we were able to characterize two additional peanut $L O X$ alleles, both repressed by Aspergillus spp. The two peanut $L O X$ alleles showed very high similarity to each other, thus indicating that they are possible allelic variants of the same locus in the allotetraploid peanut genome. To accurately determine the nature of the products that PnLOX2-3 produce, their proteins were purified and reacted

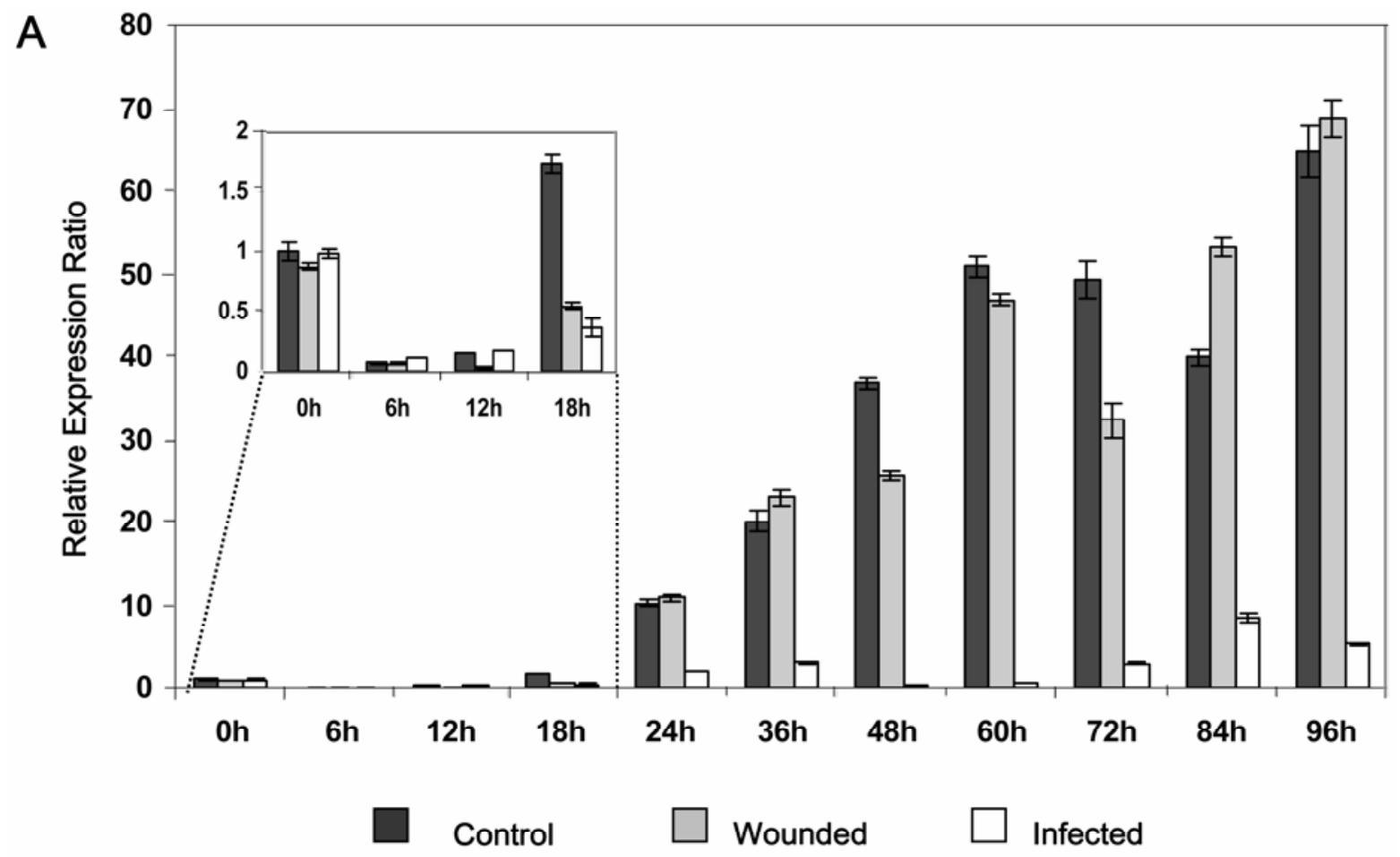

B

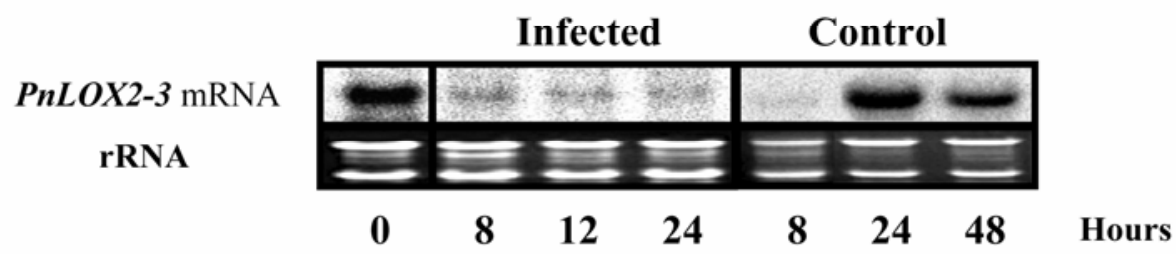

Fig. 2. PnLOX2 and PnLOX3 are repressed after Aspergillus flavus infection as revealed by A, real-time quantitative reverse-transcriptase polymerase chain reaction analysis and $\mathbf{B}$, Northern analysis $\mathbf{A}$, The transcript level is represented as the relative quantification of $P n L O X 2-3$ expression level at each timepoint, and was determined by first normalizing the target RNA to the internal standard RNA (actin), after which the normalized lipoxygenase ( $L O X$ ) RNA value was divided by the calibrator, consisting of the average of the normalized values of control (mock inoculated) samples at time 0 . The average and the standard errors were calculated for each set of six replicate reactions. B, Total RNA was extracted at the indicated timepoints and $10 \mu \mathrm{g}$ was separated on a $1.2 \%$ formaldehyde-agarose gel. Ethidium bromide-stained rRNA is indicated as a loading control. 
with linoleic acid. The purified PnLOX2 and PnLOX3 enzymes were active at a neutral to alkaline $\mathrm{pH}$ and high-performance liquid chromatography (HPLC) analysis showed that they both are predominantly $13 S$-HPODE producers.

Expression analysis of the organ specificity of PnLOX2-3 showed that the genes are highly expressed in embryo and mature cotyledons, a possible indication of involvement in seed germination or in defense mechanisms against seed pathogens. In contrast, PnLOX1 is expressed in immature seed, strongly expressed in flowers and, to some degree, in young roots, suggesting differential roles among the three peanut LOXs in plant development (Burow et al. 2000). Lipoxygenases that are involved in early stages of cotyledon development also have been described in bean (Eiben and Slusarenko 1994), young maize embryos (Jensen et al. 1997), rice (Mizuno et al. 2003), hazelnut (Santino et al. 2003), and almond (Mita et al. 2001), as well as in nitrogen-fixing nodules (Gardner et al. 1996). However, the role played by LOX in young dividing tissues is not obvious. Other researchers (Mita et al. 2001; Santino et al. 2003) have speculated that the accumulation of LOX activity in developing seed could be associated with the intense mitotic activity and the consequent rapid remodeling of cell membranes which occurs in developing seed.

Transcript analysis of infected seed showed a clear repression of PnLOX2-3 expression. This suppression of 13-LOX genes supports the previous observations that 13-HPODE content decreases during Aspergillus colonization (Burow et al. 2000) and aflatoxin development (Lopez et al. 1998). Additionally, Aspergillus infections induce the expression of a 9$L O X$ gene in maize seed and, presumably, in peanut seed based on biochemical data (Burow et al. 2000; Wilson et al. 2001). PnLOX2-3 were similarly upregulated in control and wound treatments; therefore, the genes appeared not to be wound responsive. These observations are in contrast to $P n L O X 1$, which was strongly induced by wounding and infection and was not expressed in control seed. Despite the expression of PnLOXI in infected seed, the encoding protein does not appear to contribute significantly to the oxylipin pools because $13 S$-HPODE (the major component of this LOX as determined in expression assays) content significantly decreases in Aspergillus spp.colonized seed, which we postulate is the result of PnLOX2-3 repression.
The biological function of high PnLOX2-3 activity in maturing seed is unclear; however, we hypothesize that 13-HPODEs or their products are required for normal seed development or germination. A similar role has been observed for the PTLOX3 in potato tuber development (Kolomiets et al. 2001). The multiplicity and differential regulation of $L O X$ isoforms is common in many plant species suggestive of various biological roles of these enzymes (Jalloul et al. 2002; Kolomiets et al. 2001; Royo et al. 1996; Rusterucci et al. 1999).

Our identification of Aspergillus repression of two predominately $13 S$-HPODE-producing LOX alleles in mature peanut seed complements our previous study showing a decrease of this oxylipin in infected but not healthy seed (Burow et al. 2000). We propose that a peanut 9-LOX, not yet characterized, is induced in infected seed, similar to what is observed in aflatoxin-susceptible maize lines (Wilson et al. 2001). The concomitant repression of 13-LOX and induction of 9-LOX would lead to an overall increase in the aflatoxin-stimulating oxylipin $(9 S)$ and decrease in the aflatoxin-repressive oxylipin $(13 S)$ (Fig. 3). Our hypothesis is that these seed oxylipins affect aflatoxin biosynthesis and induce Aspergillus sporulation because they have structural and biosynthetic similarities to endogenous Aspergillus oxylipins (called psi factor) that regulate toxin and sporulation processes (Tsitsigiannis et al. 2004a and b; 2005; D. I. Tsitsigiannis and N. P. Keller, unpublished data).

\section{MATERIALS AND METHODS}

\section{Plant material and seed treatments.}

The peanut (Arachis hypogaea) seed used in this study were cv. Florunner (provided by J. Starr, Department of Plant Pathology and Microbiology, Texas A\&M University, College Station), a commercial line highly susceptible to Aspergillus flavus in the field. For the peanut organ expression analysis, tissues were collected from plants cultivated in a growth chamber at 24 to $26^{\circ} \mathrm{C}$ ( $14 \mathrm{~h}$ of light and $10 \mathrm{~h}$ of dark). Samples were collected at different growth stages depending on the examined tissue, frozen in liquid nitrogen, and kept at $-80^{\circ} \mathrm{C}$ until the time of analysis.

For all seed infection studies, only the mature cotyledons were used, as has been described previously (Burow et al. 2000). For all studies, cotyledons were surface sterilized in

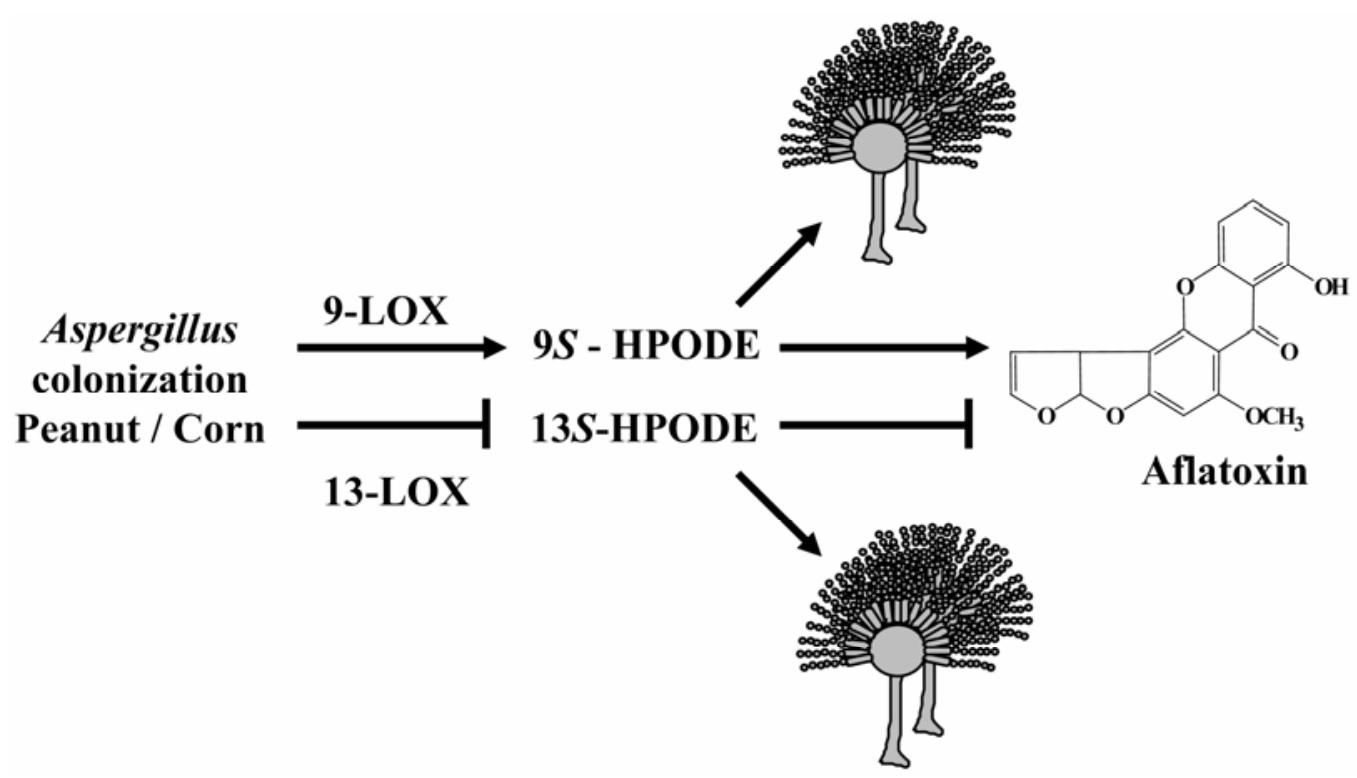

Fig. 3. Model depicting the role of Aspergillus seed colonization in regulation of 13 and 9 lipoxygenases and their derivatives as molecules modulating mycotoxin biosynthesis and fungal sporulation. 
$0.05 \%$ sodium hypochlorite for $3 \mathrm{~min}$, washed with sterile distilled water for $30 \mathrm{~s}$, followed by a wash with $70 \%$ for $5 \mathrm{~s}$ ethanol and one more wash for $30 \mathrm{~s}$ with sterile distilled water while shaking. To determine the $L O X$ expression, cotyledon treatments included water control (mock inoculation) and wounding or infection with A. flavus. For wounding experiments, cotyledons were scratched four times with a razor blade to a depth of approximately 2 to $3 \mathrm{~mm}$. For infection studies, peanut cotyledons were inoculated with A. flavus strain $12 \mathrm{~S}$ (provided by P. Cotty, United States Department of Agriculture-Agricultural Research Service, New Orleans, LA) at a concentration of $10^{5} \mathrm{conidia} / \mathrm{ml}$. A. flavus conidia were produced on potato dextrose agar media. For all treatments, 20 cotyledons were immersed in $20 \mathrm{ml}$ of sterile distilled water (control and wounded) or sterile water with A. flavus conidia in 50-ml Falcon tubes while shaking for $30 \mathrm{~min}$ in a rotary shaker $(50 \mathrm{rpm})$. All cotyledons were incubated in the dark at $29^{\circ} \mathrm{C}$ in glass petri dishes lined with three pieces of moist filter paper and a water reservoir to maintain high humidity in the artificial chamber. Samples for lipoxygenase expression analysis were collected at indicated timepoints over a time course of 4 days, by which time cotyledons were fully covered by A. flavus. Samples were frozen in liquid nitrogen and kept at $-80^{\circ} \mathrm{C}$ until the time of analysis.

\section{Isolation and cloning of PnLOX2 and PnLOX3 cDNAs.}

For total RNA extraction, the plant RNeasy kit (Qiagen, Valencia, CA, U.S.A.) was used. Briefly, 80 to $100 \mathrm{mg}$ of tissue was macerated in liquid nitrogen using a mortar and pestle, transferred quickly to a 2-ml tube, and vortexed vigorously with $600 \mu \mathrm{l}$ of RLT (kit buffer), including $10 \mu \mathrm{l}$ of $\beta$-mercaptoethanol per $1 \mathrm{ml}$ of buffer. Next steps were performed according to the manufacturer's recommendations with the modification of incubating the samples for $5 \mathrm{~min}$ in Buffer RW1 (washing step).

All the primers used in this study are shown in Table 4. For the cloning of lipoxygenase genes, a first-strand cDNA pool was generated using the Superscript pre-amplification kit (Gibco-BRL, Gaithersburg, MD, U.S.A.). Briefly, $5 \mu \mathrm{g}$ of total RNA from 8 or $48 \mathrm{~h}$ and control or infected mature cotyledons was used as template for first-strand cDNA synthesis using the oligo-dT primer and the Superscript II reverse transcriptase provided in the kit. Degenerative primers DTPNf1 and DTPNr1 were designed based on multiple amino acid and nucleotide alignment of several plant $L O X$ genes. DTPNf1 and DTPNr1 primers and the proofreading polymerase Pfu (Stratagene, La Jolla, CA, U.S.A.) used to amplify a 1.5 -kb fragment from a control or A. flavus-infected cDNA pool of a mixture of 8 - plus 48 -h treatments. The obtained PCR products were subcloned into the EcoRV site of the plasmid vector $\mathrm{pBSK}^{-}$, and 130 clones (100 from control and 30 from infected) were analyzed further to identify putative different sequences. PCR products were re-amplified from the individual plasmids, digested with Sau3A1 or AluI, and fractionated on a $2 \%$ agarose gel to further identify differential restriction patterns among the clones. Using the sequence data of representative clones, specific primers were designed and 5'-RACE and 3'-RACE technology was used to amplify the cDNA ends and obtain the full-length cDNA of putative novel peanut $L O X$ genes. Briefly, mRNA was purified using the Oligotex mRNA Isolation kit (Qiagen) from $10 \mu \mathrm{g}$ of 8or 48-h total peanut seed RNA. cDNA was synthesized from the combined 8-plus 48-h mRNA from either control or infected treatments using RACE technology (Gene Racer Kit; Invitrogen Co., Carlsbad, CA, U.S.A.) and Pfu Turbo polymerase (Stratagene) following the supplier's protocols. Fulllength sequences were obtained successfully from the sequences of group B (PnLOX2) from control cDNA and of group $\mathrm{C}(P n L O X 3)$ from infected cDNA (Table 1). The approximately 2,100-bp 3' end fragment of PnLOX2 was obtained using the forward primer $\mathrm{C} 11-\mathrm{F} 2$ and the provided reverse GeneRacer 3' primer and was subcloned into the TOPO vector (Zero Blunt TOPO PCR cloning kit, Invitrogen Co.), yielding the plasmid pDIT15.4. The approximately 1,100-bp 5 ' end fragment of PnLOX2 was obtained using the provided forward GeneRacer 5' primer and the reverse primer C11-R2 and was subcloned into the TOPO vector, yielding the plasmid pDIT14.10. Similarly, the approximately 1,700-bp 3' end fragment of PnLOX3 was obtained using the forward primer I18-F3 and the GeneRacer 3 ' primer and was subcloned into the TOPO vector, yielding the plasmid pDIT13.5. The approximately 1,500-bp 5' end fragment of PnLOX3 was obtained using the GeneRacer $5^{\prime}$ primer and the reverse primer I18-R1 and was subcloned into the TOPO vector, yielding the plasmid pDIT12.6.

\section{Expression of PnLOX2 and PnLOX3 cDNA in Escherichia coli.}

The full-length sequences of $P n L O X 2$ and $P n L O X 3$ were cloned into pET-30a vector (Novagene, Madison, WI, U.S.A.) in two steps for each construct. First, the 750-bp fragment containing the 5' end of PnLOX2 was amplified from pDIT14.10 with primers LOX2-3-F1 (NcoI) at the start codon and LOX2$\mathrm{R} 2(E c o \mathrm{RV})$ and ligated into the NcoI-EcoRV restriction sites

Table 4. Primers used in this study

\begin{tabular}{|c|c|c|}
\hline Primer & Sequence $\left(5^{\prime} \text { to } 3^{\prime} \text { end }\right)^{a}$ & Restriction enzyme site \\
\hline DTPNf1 & GAGG(ATG)GATGGAA(AC)(AGCT)GG(AGC)GAGCG & $\ldots$ \\
\hline DTPNr1 & CTT(AT)(GT)GCCACCATGGCT(CT)(AG)T(CT)T & $\ldots$ \\
\hline C11-F2 & TTTCССTTACCCTCGCAGGGGAAGAA & $\ldots$ \\
\hline C11-R2 & TTGATGACAGGTAAGGGGCTAATTG & $\ldots$ \\
\hline I18-F3 & TCGCAAGAGAAATGATTGCTGGTGTA & $\ldots$ \\
\hline I18-R1 & GCTTTTCGGAGGAAACTCTTGAAGA & $\ldots$ \\
\hline LOX2-3-F1 & TCAAACTTCAACCATGGTTTCAGGGGTA & NcoI \\
\hline LOX2-R2 & ATTTCTTGCAGGATATCTACCAGTT & EcoRV \\
\hline LOX2-F2 & 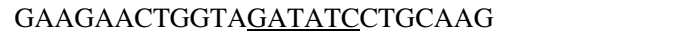 & EcoRV \\
\hline LOX2-3-R1 & AACCAGAACAGATTACTCGAGTATAATGTAGC & XhoI \\
\hline LOX3-R2 & ATAGATTGTGGGATCCAAATTGCTT & BamHI \\
\hline LOX3-F2 & AAAGCAATTTGGATCCCACAATCTA & BamHI \\
\hline RT-PnLOX2-3-F1 & ССТСАТССТССТССТТСТТС & $\ldots$ \\
\hline RT-PnLOX2-3-R1 & AAGGTGTCAACGTCCAGG & $\ldots$ \\
\hline RT-actin-F1 & GAGGAGAAGCAGAAGCAAGTTG & $\ldots$ \\
\hline RT-actin-R1 & AGACAGCATATCGGCACTCATC & $\ldots$ \\
\hline
\end{tabular}

${ }^{a}$ Underlined sequences show the restriction enzyme site in the corresponding primer. 
of pET-30a, yielding the vector pTMK9.12. Next, the 1.9-kb fragment containing the $3^{\prime}$ end of PnLOX2 was amplified from pDIT15.4 using the primers LOX2-F2 (EcoRV) and LOX2-3$\mathrm{R} 1$ (XhoI), located at $40 \mathrm{bp}$ downstream of the stop codon, and ligated into the EcoRV-XhoI sites of pTMK9.12, yielding the vector pTMK11.18. In the case of PnLOX3, first, the $1.2-\mathrm{kb}$ fragment containing the $5^{\prime}$ end of $P n L O X 3$ was amplified from pDIT12.6 with primers LOX2-3-F1 (NcoI) at the start codon and LOX3-R2 (BamHI) and ligated into the NcoI-BamHI restriction sites of pET-30a, yielding the vector pTMK10.3. Next, the $1.5-\mathrm{kb}$ fragment containing the $3^{\prime}$ end of PnLOX3 was amplified from pDIT13.5 using the primers LOX3-F2 $($ BamHI) and LOX2-3-R1 (XhoI) and ligated into the BamHIXhoI sites of pTMK10.3, yielding the vector pTMK12.6. Plasmids pTMK11.18 and pTMK12.6 encode the full-length $P n L O X 2$ and PnLOX3 coding regions, respectively, in frame with the pET-30a start site, preceded by the oligomeric histidine purification tag. All the amplifications were performed with Pfu Turbo polymerase (Stratagene).

\section{Analysis of oxidation products of PnLOX2 and PnLOX3.}

Plasmids pTMK11.18 and pTMK12.6 were used to transform Escherichia coli Rosetta cells (pLys S; Novagene). Two independent recombinants of each LOX were grown overnight at $37^{\circ} \mathrm{C}$ in $20 \mathrm{ml}$ of Luria-Bertani medium containing ampicillin at $50 \mu \mathrm{g} / \mathrm{ml}$ and kanamycin at $25 \mu \mathrm{g} / \mathrm{ml}$. This culture $(4 \mathrm{ml})$ was added to $200 \mathrm{ml}$ of the same medium. After growth at $37^{\circ} \mathrm{C}$ up to a cell density of $\mathrm{A}_{600}=0.7$, the culture was supplemented with $1 \mathrm{mM}$ isopropyl- $\beta$-D-thiogalactoside at $15^{\circ} \mathrm{C}$ for $48 \mathrm{~h}$. Cells of $200 \mathrm{ml}$ of culture were collected, suspended in $30 \mathrm{ml}$ of lysis buffer $(50 \mathrm{mM}$ Tris- $\mathrm{HCl}, \mathrm{pH} 7.5$, containing $10 \%$ [vol/vol] glycerol, $0.1 \%$ Tween $20,0.5 \mathrm{M} \mathrm{NaCl}$ ), and then disrupted using a sonifier tip with five pulses each of $30 \mathrm{~s}$. The cellular debris was removed by centrifugation $(12,000 \times g$ for $15 \mathrm{~min}$ ). The supernatant was divided into aliquots of $1 \mathrm{ml}$ and kept at $-20^{\circ} \mathrm{C}$ till it was used for further experiments (Feussner et al. 1998).

Oxygenation of linoleic acid was carried out by incubating 1 $\mathrm{ml}$ of the lysed E. coli cells expressing either PnLOX2 or PnLOX3 with the substrate $(120 \mu \mathrm{M}$ final concentration) diluted in $1 \mathrm{ml}$ of $0.1 \mathrm{M}$ sodium phosphate buffer ( $\mathrm{pH} 5.7$ to 7.04 ) or in $0.1 \mathrm{M}$ Tris buffer ( $\mathrm{pH} 7.7$ to 8.56), respectively, for $20 \mathrm{~min}$ at room temperature. The reaction products were reduced to the corresponding hydroxy fatty acids by adding $1 \mathrm{mM} \mathrm{SnCl}$ solved in $2 \mathrm{ml}$ of methanol and the reaction was stopped with $100 \mu \mathrm{l}$ of glacial acid. Lipids were extracted by adding $2 \mathrm{ml}$ of chloroform and $100 \mu \mathrm{l}$ of glacial acid. After vigorous mixing, the aqueous and the organic phase were separated by centrifugation. After recovery of the organic phase, solvents were evaporated by vacuo and the lipids were reconstituted in 0.1 $\mathrm{ml}$ of HPLC solvent.

HPLC analysis was carried out on an Agilent (Waldbronn, Germany) 1100 HPLC system coupled to a diode array detector. The absorbances at $234 \mathrm{~nm}$ (conjugated diene system of the hydroxy fatty acids) and $210 \mathrm{~nm}$ (polyenoic fatty acids) were recorded simultaneously. Straight-phase (SP)-HPLC of hydroxy fatty acid isomers was carried out on a Phenomenex Luna Silica column (Aschaffenburg, Germany; 50 by $4.6 \mathrm{~mm}$, 3- $\mu \mathrm{m}$ particle size) with a solvent system of n-hexane:2-propanol:trifluoro acetic acid (100:1:0.02, by volume) and a flow rate of $0.4 \mathrm{ml} / \mathrm{min}$. The enantiomer composition of the hydroxy fatty acids was analyzed by chiral phase-HPLC on a Chiralcel OD-H column (Diacel Chemical Industries, distributed by Merck, Darmstadt, Germany) (150 by $2.1 \mathrm{~mm}, 5-\mu \mathrm{m}$ particle size) with a solvent system of hexane:2-propanol:trifluoro acetic acid (100:5:0.02, by volume) and a flow rate of $0.1 \mathrm{ml} / \mathrm{min}$ (Feussner and Kuhn 1995).
Expression analysis using real-time qRT-PCR.

Plant RNA isolation and cDNA synthesis. Expression of the peanut $L O X$ genes in seed and different organs was examined by extracting total RNA using the RNeasy Plant Mini kit as described previously. Samples were treated with Turbo DNAfree (Ambion, Inc., Austin, TX, U.S.A.) prior to reverse transcription to remove residual amounts of DNA. Total RNA was quantified using the RiboGreen kit (Molecular Probes, Eugene, OR, U.S.A.). cDNA was generated from $1 \mu \mathrm{g}$ of total RNA using the iScript cDNA synthesis kit (Bio-Rad Laboratories, Inc., Hercules, CA, U.S.A.). The absence of DNA from the RNA samples was confirmed by performing real-time PCR on $50 \mathrm{ng}$ of total RNA (no cDNA control).

Real-time qRT-PCR primer design. The primers were designed based on the obtained PnLOX2 or PnLOX3 cDNA sequences and an expressed-sequence tag sequence of the housekeeping gene "actin depolymerizing factor" (actin-DF) (provided by P. Ozias-Akins, Department of Horticulture, University of Georgia, Tifton, U.S.A.) using the Beacon Designer software (Premier Biosoft International, Palo Alto, CA, U.S.A.) (the "Avoid Template Structure" option was selected). Peanut LOX alleles RT-PnLOX2-3-F1 and RT-PnLOX2-3-R1 were used to amplify the 145-bp PnLOX2 and PnLOX3 PCR fragment, and the primers RT-actin-F1 and RT-actin-R1 were used to amplify the 106-bp actin-DF PCR fragment. PnLOX2 and $P n L O X 3$ shared very high identity and it was not feasible to locate a primer sequence that potentially could differentiate the two alleles and test whether they have distinct expression patterns. For this reason, a unique set of primers able to amplify $P n L O X 2$ and $P n L O X 3$ but not $P n L O X 1$ was used in our gene expression studies. The resulting PCR product also was used in our Northern analysis (Fig. 2B). The housekeeping gene "actin-depolymerizing factor: actin-DF" was used as our reference or control gene because it was expressed uniformly throughout the time course tested and has been used previously as a control transcript (P. Ozias-Akins, personal communication). The efficiency of the primer pairs was determined on cDNA derived from peanut seed using fivefold serial dilutions of cDNA (starting from $100 \mathrm{ng}$ per reaction). Primer efficiency reactions were performed in triplicate (three wells per reaction) in a volume of $20 \mu \mathrm{l}$ using SYBRGreen reaction mix (Bio-Rad Laboratories, Inc.). Reactions were subjected to realtime qRT-PCR using the MyiQ Real-Time PCR Detection System and analyzed using the MyiQ software package (Bio-Rad Laboratories, Inc.). Primer efficiencies were determined using the formula efficiency $(E)=10^{(-1 / \text { slope })}$, with the slope determined by the MyiQ Cycler software. The actin-DF primers had an efficiency of 2.17 and the $L O X$ primers had an efficiency of 2.05. Sequencing of selected generated products verified that the primers were amplifying the expected $L O X$ or actin gene fragment.

Real-time $q R T-P C R$ conditions. Samples were analyzed in a volume of $20 \mu \mathrm{l}$ using iQ SYBRGreen Supermix (Bio-Rad Laboratories, Inc.). Reactions were performed in triplicate using cDNA templates from two independent iScript reactions for each gene ( $L O X$ and actin), giving a total of six replicates for each RNA sample. A master mix of SYBRGreen and primers was prepared for each primer pair. Each reaction contained $50 \mathrm{ng}$ of cDNA and $200 \mathrm{nmol}$ of each primer. Reactions were performed with the MyiQ Real-Time PCR detection system (Bio-Rad Laboratories, Inc.) using the "2-step amplification plus melting curve" protocol: $95^{\circ} \mathrm{C}$ for $3 \mathrm{~min}$ followed by 40 cycles of $95^{\circ} \mathrm{C}$ for $1 \mathrm{~min}$. (denaturation) and $55^{\circ} \mathrm{C}$ for $45 \mathrm{~s}$ (annealing and elongation). The melt curve analysis was carried out after amplification $\left(1 \mathrm{~min}\right.$ at $55^{\circ} \mathrm{C}$ followed by 8010 -s steps with a $0.5^{\circ} \mathrm{C}$ increase in temperature at each step). The determination of the threshold values $\left(\mathrm{C}_{\mathrm{t}}\right)$ was generated auto- 
matically by the MyiQ software. The identities of the amplicons and the specificity of the reactions (absence of primerdimers) were confirmed by the melt curve profile of the amplified products and agarose gel electrophoresis.

Data analysis. For the expression of the LOX genes over time after infection or in each plant tissue, the amount of target RNA ( $L O X$ RNA) at each timepoint, was determined by first normalizing the target RNA to the internal standard RNA (actin) using the $2^{-\Delta \mathrm{Ct}}$ formula or $2^{\text {(Ct internal standard) - (Ct target) }}$ (Livak and Schmittgen 2001; Pfaffl 2001). To determine the relative expression ratio of the $L O X$ gene at each treatment or each plant organ, the normalized $L O X$ RNA value was divided by the calibrator value. For determining the relative expression in cotyledons, the calibrator was the average of the normalized values of control (mock-inoculated) samples at time 0 . For the comparison of $L O X \mathrm{mRNA}$ levels in different plant tissues, we chose the average of the normalized values of flower RNA samples as the calibrator. The average, standard error, and coefficient of variation were determined for each set of six replicate reactions. The entire analysis was replicated in three independent experiments for the timepoints $6,12,48$, and $84 \mathrm{~h}$.

\section{ACKNOWLEDGMENTS}

This work was supported by International Crops Research Institute for the Semi-Arid Tropics to N. P. Keller and the Dr. George Washington Carver award to D. I. Tsitsigiannis by the United States National Peanut Board. We are grateful to P. Ozias-Akins (University of Georgia) for providing the sequence of the actin-DF, J. Starr (Texas A\&M University) for providing the peanut seed, and T. Kowieski (University of WisconsinMadison) for experimental help.

\section{LITERATURE CITED}

Bell, E., and Mullet, J. E. 1993. Characterization of an Arabidopsis lipoxygenase gene responsive to methyl jasmonate and wounding. Plant Physiol. 103:1133-1137.

Bennett, J. W., and Klich, M.. 2003. Mycotoxins. Clin. Microbiol. Rev. $16: 497-516$

Blee, E. 2002. Impact of phyto-oxylipins in plant defense. Trends Plant Sci. 7:315-22

Burow, G. B., Nesbitt, T. C., Dunlap, J. and Keller, N. P. 1997. Seed lipoxygenase products modulate Aspergillus mycotoxin biosynthesis. Mol. Plant-Microbe Interact. 10:380-387.

Burow, G. B., Gardner, H. W., and Keller, N. P. 2000. A peanut seed lipoxygenase responsive to Aspergillus colonization. Plant Mol. Biol. 42:689-701.

Calvo, A. M., Hinze, L. L., Gardner, H. W., and Keller, N. P. 1999. Sporogenic effect of polyunsaturated fatty acids on development of Aspergillus spp. Appl. Environ. Microbiol. 65:3668-3673.

Choi, D., Bostock, R. M., Avdiushko, S, and Hildebrand, D. F. 1994 Lipid-derived signals that discriminate wound- and pathogen-responsive isoprenoid pathways in plants: Methyl jasmonate and the fungal elicitor arachidonic acid induce different 3-hydroxy-3-methylglutarylcoenzyme A reductase genes and antimicrobial isoprenoids in Solanum tuberosum L. Proc. Natl. Acad. Sci. U.S.A. 91:2329-2333.

Cleveland, T. E., Dowd, P. F., Desjardins, A. E., Bhatnagar, D., and Cotty, P. J. 2003. United States Department of Agriculture-Agricultural Research Service research on pre-harvest prevention of mycotoxins and mycotoxigenic fungi in U.S. crops. Pest Manage. Sci. 59:629-642.

Doehlert, D. C., Wicklow, D. T., and Gardner, H. W. 1993. Evidence implicating the lipoxygenase pathway in providing resistance to soybean against Aspergillus flavus. Phytopathology 183:1473-1477.

Eiben, H. G., and Slusarenko A. J. 1994. Complex spatial and temporal expression of lipoxygenase genes during Phaseolus vulgaris (L.) development. Plant J. 5:123-135.

Farmer, E. E., Almeras, E., and Krishnamurthy, V. 2003. Jasmonates and related oxylipins in plant responses to pathogenesis and herbivory. Curr. Opin. Plant Biol. 6:372-378.

Feussner, I., and Kuhn, H. 1995. The lipid body lipoxygenase from cucumber seedlings exhibits unusual reaction specificity. FEBS (Fed. Eur. Biol. Soc.) Lett. 367:12-14.

Feussner, I., and Wasternack, C. 2002. The lipoxygenase pathway. Annu. Rev. Plant Physiol. Plant Mol. Biol. 53:275-297.
Feussner, I., Bachmann, A., Hohne, M., and Kindl, H. 1998. All three acyl moieties of trilinolein are efficiently oxygenated by recombinant Histagged lipid body lipoxygenase in vitro. FEBS (Fed. Eur. Biol. Soc.) Lett. 431:433-436.

Gardner, C. D., Sherrier, D. J., Kardailsky, I. V., and Brewin, N. J. 1996 Localization of lipoxygenase proteins and mRNA in pea nodules: Identification of lipoxygenase in the lumen of infection threads. Mol. PlantMicrobe Interact. 8:282-289.

Gobel, C., Feussner, I., and Rosahl, S. 2003. Lipid peroxidation during the hypersensitive response in potato in the absence of 9-lipoxygenases. $\mathrm{J}$. Biol. Chem. 278:52834-52840.

Goodrich-Tanrikulu, M., Mahoney, N. E., and Rodriguez, S. B. 1995. The plant growth regulator methyl jasmonate inhibits aflatoxin production by Aspergillus flavus. Microbiology 141:2831-2837.

Hildebrand, D. F., Fukushige, H., Afitlhile, M., and Wang, C. 1998. Lipoxygenases in plant development and senescence. Pages 151-181 in: Eicosanoids and Related Compounds in Plants and Animals. A. F. Rowley, H. Kuhn, and T. Schewe, eds. Princeton University Press, Princeton, NJ, U.S.A.

Howe, G. A., and Schilmiller, A. L. 2002. Oxylipin metabolism in response to stress. Curr. Opin. Plant Biol. 5:230-236.

Jalloul, A., Montillet, J. L., Assigbetse, K., Agnel, J. P., Delannoy, E., Triantaphylides, C., Daniel, J. F., Marmey, P., Geiger, J. P., and Nicole, M. 2002. Lipid peroxidation in cotton: Xanthomonas interactions and the role of lipoxygenases during the hypersensitive reaction. Plant $\mathrm{J}$. 32:1-12.

Jensen, A. B., Poca, E., Rigaud, M., Freyssinet, and Pages, M. 1997. Molecular characterization of L2 lipoxygenase from maize embryos. Plant Mol. Biol. 33:605-614

Kolomiets, M. V., Chen, H., Gladon, R. J., Braun, E. J., and Hannapel, D. J. 2000. A leaf lipoxygenase of potato induced specifically by pathogen infection. Plant Physiol. 124:1121-1130.

Kolomiets, M. V., Hannapel, D. J., Chen, H., Tymeson, M., and Gladon, R. J. 2001. Lipoxygenase is involved in the control of potato tuber development. Plant Cell 13:613-626.

Livak, K. J., and Schmittgen, T. D. 2001. Analysis of relative gene expression data using real-time quantitative PCR and the 2(-delta delta C(T) Method. Methods 25:402-408.

Lopez, Y., Keller, N.P., Sarr, B., Phillips, T. D., Cuero, R. G., and Smith, O. D. 1998. Visual estimation of aflatoxin production in peanut with Aspergillus norsolorinic acid mutants. Peanut Sci. 25:93-99.

Mita, G., Gallo, A., Greco, V., Zasiura, C., Casey, R., Zacheo, G., and Santino, A. 2001. Molecular cloning and biochemical characterization of a lipoxygenase in almond (Prunus dulcis) seed. Eur. J. Biochem. 268:1500-1507.

Mizuno, K., Iida, T., Takano, A., Yokoyama, M., and Fujimura, T. 2003. A new 9-lipoxygenase cDNA from developing rice seeds. Plant Cell Physiol. 44:1168-1175

Oh, K. B., Miyazawa, H., Naito, T., and Matsuoka, H. 2001. Purification and characterization of an autoregulatory substance capable of regulating the morphological transition in Candida albicans. Proc. Natl. Acad. Sci. U.S.A. 98:4664-4668.

Pattee, H. E., and Singleton, J. A. 1977. Isolation of isomeric hydroperoxides from the peanut lipoxygenase-linoleic acid reaction. J. Am. Oil Chem. Soc. 54:183-185.

Pfaffl, M. W. 2001. A new mathematical model for relative quantification in real-time RT-PCR. Nucleic Acids Res. 29:e45.

Porta, H., and Rocha-Sosa, M. 2002. Plant lipoxygenases. Physiological and molecular features. Plant Physiol. 130:15-21.

Rance, I. I., Fournier, J., and Esquerre-Tugaye, M. T. 1998. The incompatible interaction between Phytophthora parasitica var. nicotianae race 0 and tobacco is suppressed in transgenic plants expressing antisense lipoxygenase sequences. Proc. Natl. Acad. Sci. U.S.A. 95:6554-6559.

Royo, J., Vancanneyt, G., Perez, A. G., Sanz, C., Stormann, K., Rosahl, S., and Sanchez-Serrano, J. J. 1996. Characterization of three potato lipoxygenases with distinct enzymatic activities and different organ-specific and wound-regulated expression patterns. J. Biol. Chem. 271:21012-21019.

Royo, J., Leon, J., Vancanneyt, G., Albar, J. P., Rosahl, S., Ortego, F., Castanera, P., and Sanchez-Serrano, J. J. 1999. Antisense-mediated depletion of a potato lipoxygenase reduces wound induction of proteinase inhibitors and increases weight gain of insect pests. Proc. Natl. Acad. Sci. U.S.A. 96:1146-1151.

Rusterucci, C., Montillet, J. L., Agnel, J. P., Battesti, C., Alonso, B., Knoll, A., Bessoule, J. J., Etienne, P., Suty, L., Blein, J. P., and Triantaphylides, C. 1999. Involvement of lipoxygenase-dependent production of fatty acid hydroperoxides in the development of the hypersensitive cell death induced by cryptogein on tobacco leaves. J. Biol. Chem. 274:36446-36455.

Sanders, T. H., Pattee, H. E., and Singleton, J. A. 1975. Lipoxygenase isozymes of peanut. Lipids 10:681-685. 
Santino, A., De Paolis, A., Gallo, A., Quarta, A., Casey, R., and Mita, G. 2003. Biochemical and molecular characterization of hazelnut (Corylus avellana) seed lipoxygenases. Eur. J. Biochem. 270:4365-4375.

Shibata, D., Slusarenko, A., Casey, R., Hildebrand, D., and Bell, E. 1994. Lipoxygenases. Plant Mol. Biol. Rep. 12:S41-42.

Steczko, J., Donoho, G. P., Clemens, J. C., Dixon, J. E., and Axelrod, B. 1992. Conserved histidine residues in soybean lipoxygenase: Functional consequences of their replacement. Biochemistry 31:4053-4057.

Tsitsigiannis, D. I., Kowieski, T. M., Zarnowski, R. and Keller, N. P. 2004a. Lipogenic signals act as developmental regulators of spore balance in Aspergillus nidulans. Eukaryotic Cell 3:1398-1411.

Tsitsigiannis, D. I., Kowieski, T. M., Zarnowski, R. and Keller, N. P. 2005. Three putative oxylipin biosynthetic genes integrate sexual and asexual development in Aspergillus nidulans. Microbiology 151:18091821.

Tsitsigiannis, D. I., Zarnowski, R., and Keller, N. P. 2004b. The lipid body protein, PpoA, coordinates sexual and asexual sporulation in Aspergillus nidulans. J. Biol. Chem. 279:11344-11353.

Vergopoulou, S., Galanopoulou, D., and Markaki, P. 2001. Methyl jas- monate stimulates aflatoxin B1 biosynthesis by Aspergillus parasiticus. J. Agric. Food Chem. 49:3494-3498.

Wang, L. H., He, Y., Gao, Y., Wu, J. E., Dong, He, C., Wang, S. X., Weng, L. X., Xu, J. L., Tay, L., Fang, R. X., and Zhang, L. H. 2004. A bacterial cell-cell communication signal with cross-kingdom structural analogues. Mol. Microbiol. 51:903-912.

Wasternack, C., and Hause, B. 2002. Jasmonates and octadecanoids: Signals in plant stress responses and development. Prog. Nucleic Acid Res. Mol. Biol. 72:165-221.

Wilson, R. A., Gardner, H. W., and Keller, N. P. 2001. Cultivar-dependent expression of a maize lipoxygenase responsive to seed infesting fungi. Mol. Plant-Microbe Interact. 14:980-987.

Wright, M. S., Greene-McDowell, D. M., Zeringue, H. J., Jr., Bhatnagar, D., and Cleveland, T. E. 2000. Effects of volatile aldehydes from Aspergillus-resistant varieties of corn on Aspergillus parasiticus growth and aflatoxin biosynthesis. Toxicon 38:1215-1223.

Zeringue, H. J. 1996. Possible involvement of lipoxygenase in a defense response in aflatoxigenic Aspergillus cotton plant interactions. Can. J. Bot. 74:98-102. 